\title{
On the measurement of solute concentrations in 2-D flow tank experiments
}

\author{
M. Konz ${ }^{1}$, P. Ackerer ${ }^{2}$, E. Meier ${ }^{3}$, P. Huggenberger ${ }^{1}$, E. Zechner ${ }^{1}$, and D. Gechter ${ }^{1}$ \\ ${ }^{1}$ University of Basel, Departement Umweltwissenschaften, Switzerland \\ ${ }^{2}$ Université Louis Pasteur, Institut de Mécanique des Fluides et des Solides, CNRS, UMR 7507,Strasbourg, France \\ ${ }^{3}$ Edi Meier \& Partner AG, Winterthur, Switzerland
}

Received: 30 October 2007 - Published in Hydrol. Earth Syst. Sci. Discuss.: 20 November 2007

Revised: 8 April 2008 - Accepted: 9 April 2008 - Published: 14 May 2008

\begin{abstract}
In this study we describe and compare photometric and resistivity measurement methodologies to determine solute concentrations in porous media flow tank experiments. The first method is the photometric method, which directly relates digitally measured intensities of a tracer dye to solute concentrations, without first converting the intensities to optical densities. This enables an effective processing of a large number of images in order to compute concentration time series at various points of the flow tank and concentration contour lines. This paper investigates perturbations of the measurements; it was found both lens flare effects and image resolution were a major source of error. Attaching a mask minimizes the lens flare. The second method for in situ measurement of salt concentrations in porous media experiments is the resistivity method. The resistivity measurement system uses two different input voltages at gilded electrode sticks to enable the measurement of salt concentrations from 0 to $300 \mathrm{~g} / \mathrm{l}$. The method is highly precise and the major perturbations are caused by temperature changes, which can be controlled in the laboratory. The two measurement approaches are compared with regard to their usefulness in providing data for benchmark experiments aimed at improving process understanding and testing numerical codes. Due to the unknown measurement volume of the electrodes, we consider the image analysis method more appropriate for intermediate scale 2D laboratory benchmark experiments for the purpose of evaluating numerical codes.
\end{abstract}

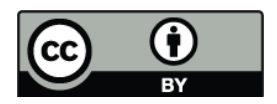

Correspondence to: $\mathrm{M}$. Konz (m.konz@gmx.de)

\section{Introduction}

Laboratory experiments are an excellent way of providing data to develop transport theories and validate numerical codes. They have several advantages: boundary and initial conditions are known, the porous medium properties can be defined separately and the experiments can be repeated if necessary. Excellent reviews of mass transfer in porous media at the laboratory scale can be found in Silliman et al. (1998) and Simmons et al. (2002). A variety of methods exists for qualitative and quantitative determination of solute transport in porous media experiments. Traditionally this is done by intrusive methods where extracted fluids are analyzed in the laboratory (e.g. Swartz and Schwartz, 1998; Barth et al., 2001). These methods are work intensive and require a well-equipped laboratory for chemical analysis. Further, the temporal and spatial resolution of concentration determination is limited due to point sampling, and perturbations of the flow field cannot be excluded. Oostrom et al. (2003) introduced the non-intrusive dual-energy gamma radiation system methods for 3D experiments. Oswald et al. (1997) and Oswald and Kinzelbach (2004) used the nuclear magnetic resonance (NMR) technique to derive concentration distributions of 3D benchmark experiments for density dependent flow. This method is very precise but it is limited to small flow tank experiments due to the size of the NMR tomograph.

In the published literature, dyes have been used mainly to qualitatively and quantitatively visualize spatial mixing patterns of contaminants in intermediate scale 2-D porous media flow tank experiments. The dye itself can be used as contaminant (e.g. Rahman et al., 2005), or it acts as an optical tracer to mark contaminants with density effects (e.g. Schincariol et

Published by Copernicus Publications on behalf of the European Geosciences Union. 
al., 1993; Swartz and Schwartz, 1998; McNeil et al., 2006; Goswami and Clement, 2007).

Reflected light is used for image analysis in most intermediate scale experiments (e.g. Oostom et al., 1992; Swartz and Schwartz, 1998, Wildenschild and Jensen, 1999; Simmons et al., 2002; Rahman et al., 2005; McNeil et al., 2006; Goswami and Clement, 2007). The advantage of this technique is that it can be used with non-transparent porous media material, e.g., sand and with thick flow tanks that prevent light transmission. However, the use of reflected light to quantify dye concentrations can lead to problems with image noise, reflected and diffusive light from surroundings and can therefore alter the image quality. A promising alternative is the light transmission technique to measure emitted light from behind the flow tank opposite the light source (e.g. Corapcioglu et al., 1997; Detwiler et al., 2000; Huang et al., 2002; Theodoropoulou et al., 2003; Jones et al., 2005). Catania et al. (2008) provide an excellent literature review of transmissive experiments and data processing techniques. These studies used very thin small-scale flow models of maximum $1 \mathrm{~cm}$ thickness, which enable light transmission.

Variations in lighting, exposure and film development result in non-uniform image intensities between successive images. Rahman et al. (2005) used the $\gamma$-calibration model to correct the color representation of images $\left(I_{\text {corr }}\right)$ :

$I_{\text {corr }}=a+b \cdot I^{\gamma}$

where $a, b$ and $\gamma$ are correction parameters, assumed to be spatially uniform, and $I$ is the measured intensity. The values of the parameters are determined by fitting the measured intensities of color cards to the ideal model. Schincariol et al. (1993) and McNeil et al. (2006), amongst others, convert the measured intensities to optical density. Optical density $D$ is non-linearly related to intensity $I$ by:

$D=\log _{10}(a / I)$

where $a$ is a constant of proportionality. This involves the optimization of intensity vs. optical density standard curve of each image to be investigated and an optimization of optical density vs. concentration standard curve. However, the conversion of intensity to optical density or the application of the $\gamma$-calibration model is only necessary if:

1. Analogue images are taken and the film has to be developed and scanned to convert the image to digital data ;

2. Automatically, non-linearly adjusted and compressed images are used.

If only the spatial evolution of plumes at a limited number of timesteps is investigated, the computational efficiency is of minor importance because the intensity vs. optical density standard curve has to be optimized only for a small number of images. For the evaluation of numerical codes however, breakthrough curves at distinct points with a high temporal resolution are necessary. This requires processing of a large number of images and the computational efficiency becomes a significant criteria. As opposed to Schincariol et al. (1993), Swartz and Schwartz (1998) and McNeil et al. (2006) we relate linearly measured intensities directly to concentrations without standardization to optical densities to enable processing of a large number of images with the aim of deriving breakthrough curves of a high temporal resolution. In this study, we apply the light reflection technique because the back wall of our flow tank holds devices for measuring resitivity and pressure, which preclude the use of a light transmission technique. The light reflection technique is more appropriate if experiments are done with porous media that is more opaque then glass beads such as clayey inclusions and lenses. Therefore, this study aims to provide details on this technique. We investigate the impact of image resolution (pixels per length) on the precision of the results, and the impact of lens flare on intensity measurements. In the literature on photometric measurements of plume distribution in flow tanks, little attention has been paid to errors in concentration determination caused by these effects.

While image analysis is a useful tool for 2-D applications, electrical resistivity measurement technique to determine salt concentrations $(0-300 \mathrm{~g} / \mathrm{l})$ at discrete points within the porous media can be used both in 2-D and 3-D applications. We present in this paper technical details of the resistivity method and discuss the implementation of this method for porous media experiments. Resistivity measurement is a well-developed technology to determine ion concentrations of fluids. However, there are only few examples in literature where this technology is used for in-situ measurements in porous media experiments. Siliman and Simpson (1987) used electrode arrays in a narrow interval of low concentrations from 50 to $1000 \mathrm{mg} / \mathrm{l}$. Hassanizadeh and Leijnse (1995) took platinum disc electrodes facing each other to measure salt concentrations in the range of 0.001 to $0.24 \mathrm{~kg} \mathrm{~kg}^{-1}$ (about $280 \mathrm{~g} / \mathrm{l}$ ). A 3-D application is given in Danquigny et al. (2004). However, none of the authors present technical details of their systems. Since not all measurement systems are standard commercial systems, a significant effort is necessary to develop the technology. The aim of the detailed description of both independent measurement techniques, resistivity measurement and image analysis is to provide experimentalists with information to construct measurement systems and assess their applicability to the specific experiments. We demonstrate the advantages and limitations of these techniques based on a model experiment, and discuss them in view of their applicability to benchmark experiments on density dependent flow. 


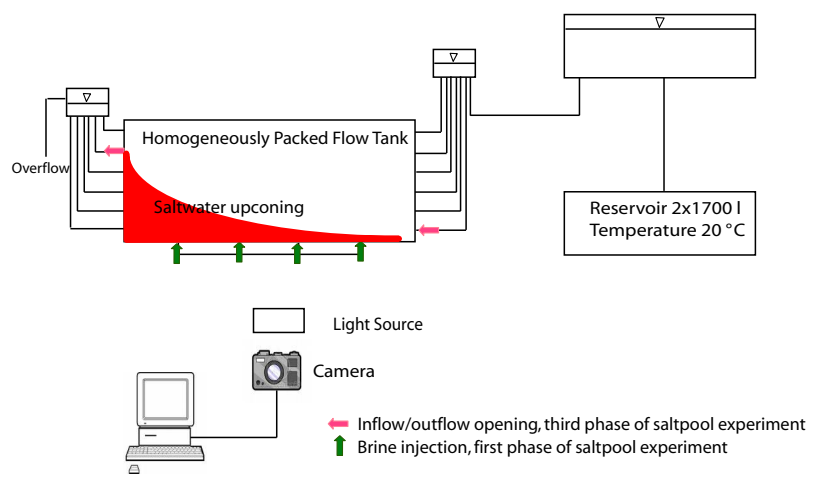

Fig. 1. Schematic experimental setup.

\section{Description of the experimental setup}

The flow experiments were conducted in a Plexiglas tank with the dimensions $(L \times H \times W)$ of $1.58 \mathrm{~m} \times 0.6 \mathrm{~m} \times 0.04 \mathrm{~m}$ (Figs. 1 and 2). The back of the tank holds measurement instruments, such as pressure sensors, electrodes of the resistivity measurement cell system (RMC) and temperature sensors. The front side of the tank consists of a clear Plexiglas pane facilitating visual observation of the tracer movement through the homogenous porous medium during the course of the experiments. The tank has four openings at the bottom, which are regulated by valves. Another six openings each are placed at the left and right side of the tank. These openings are also regulated by valves and connected to freshwater reservoirs. The height of the reservoirs can be adjusted to adapt the velocity of the water inside the porous media. Two 17001 deionized freshwater reservoirs, where the water temperature is kept constant at $20^{\circ} \mathrm{C}$ to avoid degassing in the porous media, maintain water supply to the inflow reservoir. Tension pins with a diameter of $0.5 \mathrm{~cm}$ were installed within the tank to prevent the deformation of the Plexiglas wall. Because these obstacles are minor, we assume that they do not perturb the flow. Glass beads with a diameter of $0.6 \mathrm{~mm}$ were used as a porous media. The tank was homogenously packed. During filling, there was always more water than glass beads in the tank in order to avoid air trapping. A neoprene sheet and air tubes were placed between the Plexiglas cover and the porous media. The air tubes can be inflated to compensate for the free space when the glass bead settles, preventing preferential flow along the top cover of the tank. A supplementary experiment with traced water and horizontal flow conditions revealed that there are no preferential pathways and that the water flows homogenously through the porous media.

Five different experiments were conducted in order to assess the image analysis methodology and the resistivity measurement system. Table 1 summarizes the experiments. In the first experiment E1, we successively placed black cards on the front window of the tank to demonstrate the lens flare effect (Sect. 3.5). E2 and E3 are density-dependent flow ex-

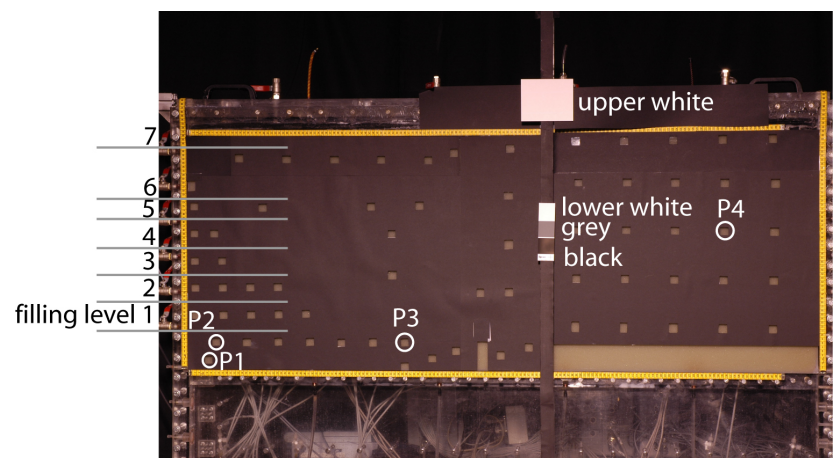

Fig. 2. Tank of E3 with mask to reduce effects of flare and filling levels of E4.

periments using red dyed saltwater with a density of $1063 \mathrm{~g} / \mathrm{l}$. The experiments E2 and E3 in Table 1 (Sect. 3.5) consisted of four phases. The brine with an initial concentration of 100 $\mathrm{g} / \mathrm{l}$ was marked with $1 \mathrm{~g} / \mathrm{l}$ of the red food dye Cochineal Red A (E124). In the first phase of the experiment, the brine was pumped into the domain from four openings at the bottom of the tank, as indicated in Fig. 1 by the green arrows. Buoyancy effects forced the brine to move laterally in the second phase and form a planar brine-freshwater interface. No flow was applied in this second phase through any openings in the tank. At the end of this phase, the brine had equilibrated with a mixing zone of about $4 \mathrm{~cm}$ above $3 \mathrm{~cm}$ of brine with the initial concentration of $100 \mathrm{~g} / \mathrm{l}$. The head difference between inflow and outflow reservoirs generated a constant flow during the third phase of the experiment, which we refer to in the following as the flow phase. Flow through the inflow opening on the lower left side of the tank $(11 \mathrm{~cm}$ above the ground, red arrow in Fig. 1), close to the brine and the outflow opening on the upper right side of the tank $(45 \mathrm{~cm}$ above the ground, red arrow in Fig. 1), forced an upconing of the saltwater below the outlet as shown in Fig. 1. Mechanical dispersion and advection processes cause the upconing of the saltwater. The last phase is the calibration phase to enable the conversion of dye intensities to concentrations, (for details on this phase see Sect. 3.4).

The difference between E2 and E3 is the attached mask in E3. The mask has observation holes and reduces the flare effect. E4 is an experiment to assess the error caused by the flare effect. We filled the tank progressively with solutions of different salt-dye concentrations and evaluated the impact of lens flare on the observation holes (see Fig. 2 for filling levels). In E5, the dense saltwater (same as in E2 and E3) is continuously pumped into the porous media through a single inflow opening at the edge of the tank. This enables the exact quantification of saltwater entering the domain and the image analysis technique can be verified using salt mass balances. 
Table 1. Experiments conducted to assess the image analysis technique and the resistivity measurement cell (RMC) technology.

\begin{tabular}{lcl}
\hline Experiment & Number & Objective \\
\hline $\begin{array}{l}\text { Successively attached black cards } \\
\text { Saltpool flow experiment; without mask }\end{array}$ & E1 & $\begin{array}{l}\text { Demonstrate lens flare effects } \\
\text { Demonstrate the impact of lens } \\
\text { flare on measured reflection intensities }\end{array}$ \\
$\begin{array}{l}\text { Saltpool flow experiment; with mask } \\
\begin{array}{l}\text { Progressively filling of salt-dye solutions } \\
\text { of different concentrations; with mask }\end{array}\end{array}$ & E3 & $\begin{array}{l}\text { Test a possibility to reduce the measurement error } \\
\text { Estimate the error caused by lens flare effects }\end{array}$ \\
$\begin{array}{l}\text { Continuous inflow of marked saltwater } \\
\text { E5 }\end{array}$ & Eass balance determination at different times
\end{tabular}

\section{Concentration determination with photometry}

3.1 Image acquisition and general concept of optical concentration determination

The dense fluid was marked with a dye to visually differentiate the saltwater from the ambient pore water. Cochineal Red A (E124) was used as tracer. This food dye is nonsorbing, nonreactive with $\mathrm{NaCl}$ in concentrations used for the experiment (Rahman et al., 2005 and our own batch experiments). Photometric analysis of different dye-saltwater solutions revealed that there is no degradation or optical decay of dye over a period of up to 7 days. Different diffusivity could cause separation of dye and saltwater leading to erroneous concentration determinations. In the literature other authors have used various dyes, including food color, to track the movement of dense saltwater in laboratory-scale aquifer models. Neither Schincariol et al. (1993), Swartz and Schwartz (1998) nor Goswami and Clement (2007) reported difficulties due to dye-salt separation. The latter used a red food color comparable to Cochineal Red A. We conducted column experiments ( $1 \mathrm{~m}$ length) with different saltdye solutions and compared breakthrough curves of salt and colour. The salt concentrations were measured using standard resistivity cells (WTW) and the color concentrations were determined using a spectrometer (Shimadzu UV-1700 Pharma Spec). No separation could be observed. Furthermore, differences in the diffusivity are of minor importance in experiments dominated by advection as is the case in our experiments.

To determine concentration distributions in the tank, we took photos with a digital camera (Nikon D70) using a reproducible illumination with a single light source placed right above the camera at a distance of $\sim 3 \mathrm{~m}$ from the tank. The light source $($ EKON JM-T $1 \times 400 \mathrm{~W}, \mathrm{E} 40)$ was adjusted and checked with a luxmeter to minimize spatial lightning nonuniformity. However, it was not possible to totally avoid lightning nonuniformity and a higher intensity remains in the center of the photographed domain. White, grey and black cards were attached on the front pane of the tank (Fig. 2). A dark curtain placed around the entire experimental setup prevented reflections of any objects on the tank pane. Furthermore, in order to avoid lighting nonuniformity due to daylight, there were no windows in the laboratory. All camera parameters were set manually (shutter speed $1 / 8 \mathrm{~s}$, aperture 13.0, ISO200). For image processing it is important that images, taken at different times, match on a pixel-bypixel basis. A computer programme (Nikon camera controlPRO) controlled the camera, which was not touched or removed during the entire experiment including the calibration procedure. Therefore, neither rotation nor translation of images is necessary. The digital camera stores linear raw data (.nef) beside the automatically non-linearly processed photos (.jpg). Raw data is the output from each of the original red, green and blue sensitive pixels of the image sensor, after being read out by the array electronics and passing through an analogue-to-digital converter. A linear development means that no gamma correction has been applied yet, and thus levels are distributed along the histogram in a linear way being each of them proportional to the amount of light (number of photons) that the pixel was able to capture during the exposure. The used converter, dcraw, generates linear 16-bit tiff images from the raw data The 12-bit information gained from the camera is linearly spread along the 16-bit of the tiff image. This was necessary because standard PC systems either work on 8- or 16-bit but not on 12- bit. 16- bit was taken to preserve the information of the 12- bit data (e.g. http: //www.guillermoluijk.com/tutorial/dcraw/index_en.htm).

The image-processing procedure includes the following steps: (1) data converting to 16 bit tiff images (65536 intensity values per channel of the RGB color space) with the freeware dcraw (http://cybercom.net/ dcoffin/dcraw/), (2) selection of green channel (most sensitive to dye concentrations), (3) correction of fluctuations in brightness, (4) determination of measurement area, (5) construction of a curve that relates intensities to concentrations from calibration images and determination of function parameters for the mathematical formulation of the curve, and (6) conversion of measured intensities of the experiment into concentrations. In the following, steps 3 to 6 are described in detail and we discuss the impact of lens flare on the measurements. 


\subsection{Correction of fluctuations in brightness (Step 3)}

A constant light source is difficult to achieve because of fluctuations in energy supply. The first four graphs in Fig. 3 show the intensities of attached white, grey and black cards (see Fig. 2 for the position of the cards), which are strongly influenced by the fluctuations in lightning. The spikes in the raw data (first four graphs) could be caused by fluctuations in electricity supply. The intensities shown in the figure were recorded over two days including the night. 0-600 were taken in the evening and overnight, whereas 600 onwards are images taken in the morning and over the day. This could explain the increased fluctuations in energy supply during the day. Existing methods of image processing convert measured intensities into optical densities based on intensity vs. optical density standard curve in order to correct fluctuations in brightness. The reference values of optical density are taken from the attached grey scales. This step is necessary if the raw data are non-linear because analogue photography is used and the photos are developed and scanned, or automatically pre-processed images are taken. Due to the linearity of our raw data, neither the $\gamma$-calibration model (Rahman et al., 2005) nor the translation to optical density (e.g. Schincariol et al., 1993) to correct fluctuations in brightness is necessary. The fluctuations can be corrected $\left(I_{\text {corr }}(i, j)\right)$ using the attached upper white card as reference value $\left(I_{\text {ref }}\right)$ for each image:

$I_{\text {corr }}(i, j)=I(i, j) / I_{\text {ref }}$

The last three graphs in Fig. 3 show how the correction method works for the lower white card, the grey and the black cards (Fig. 2). We tested this approach for several points on the tank located not only in the center of the tank but also in the corner of the domain where illumination differences between the reference white and the observation points are highest. The method performs well at all observation points. As a consequence, the images are on the same intensity level after the correction step, and changes in intensity do result only from changes in dye concentration and not from fluctuation in lightning. Minor fluctuations still persist and are related to scattered reflection on the camera lens, which cannot be corrected. These uncertainties are treated in the error estimation in Sect. 3.6.

3.3 Impact of optical heterogeneity of porous media and determination of measurement area (Step 4)

The resolution of the images is crucial, especially in experiments where concentrations vary spatially with a steep gradient, e.g., in transition zones. In the literature, little attention has been paid to analyzing the impact of the resolution on the precision of the derived concentration data. Schincariol et al. (1993) suggested a general median smoothing $(3 \times 3$ pixels $)$ to reduce noise from bead size. The image resolution of our digital images (approx. $0.31 \mathrm{~mm}^{2} /$ pixel) im-

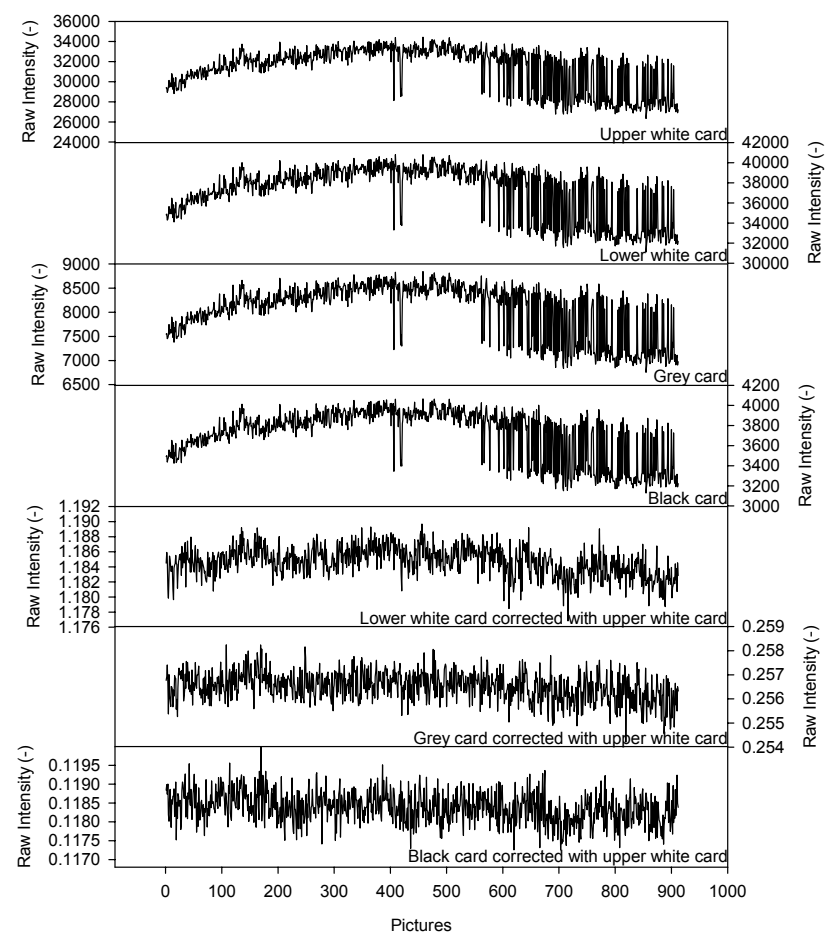

Fig. 3. Measured and processed intensity values of grey and black cards.

plies a high resolution of the concentration determination at a point. In order to assess the impact of resolution, we filled the tank with solutions of different concentrations, took images of each solution and analyzed the intensity distributions in squares of $25 \mathrm{~cm}^{2}$ (8281 pixels) at different positions of the image. The squares were filled equally with the respective solution and, due to the small extent of $25 \mathrm{~cm}^{2}$, the impact of uneven lighting can be excluded. As an example of this Fig. 4A shows the intensities of one of the squares for $100 \mathrm{~g} / \mathrm{l}$ derived from $1 \times 1$ pixel and from the median over $5 \times 5,10 \times 10$ and $18 \times 18$ pixels. The fluctuation of intensities increases with increasing resolution due to the noise from bead size or the position of the beads.

The comparison of the $95 \%$ confidential intervals in Fig. 4B shows that the precision of the medians increases with increasing edge length. These patterns apply to all concentrations and are demonstrated for $100 \mathrm{~g} / \mathrm{l}$. The calculated intensities vary significantly for resolutions smaller than $10^{2}$ pixels. In this study the median over 100 pixels (10 pixels edge length) is taken to calculate concentrations at a point. This corresponds to an area of about $31 \mathrm{~mm}^{2}$. The actual resolution for concentration determination is therefore lower than the image resolution itself. Since the noise from the grain size depends on the grain diameter, the adequate resolution has to be derived for each experimental setup. 

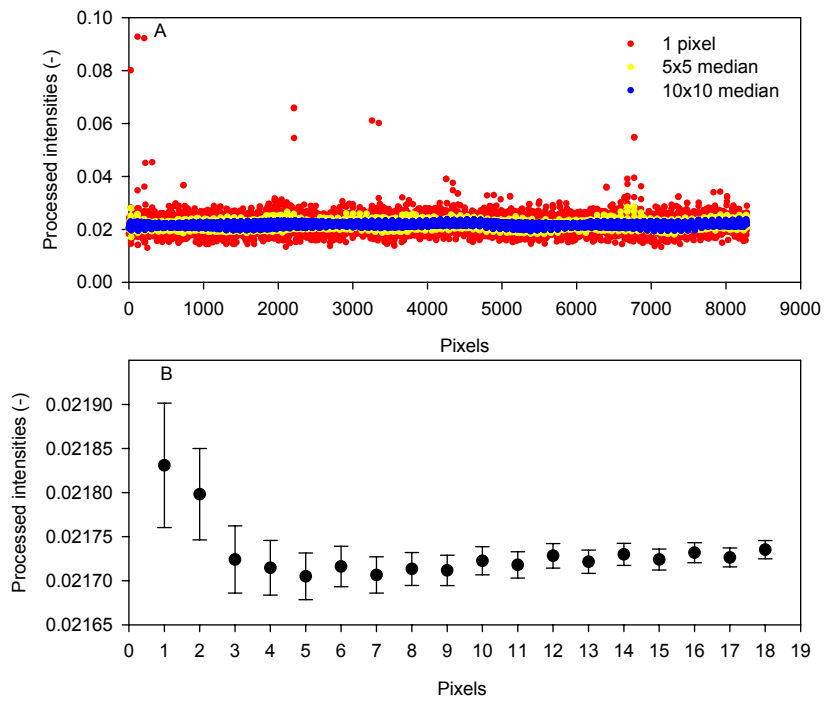

Fig. 4. (A) Intensities of the 8281 pixels of a square of $5 \times 5 \mathrm{~cm}^{2}$ of calibration image $100 \mathrm{~g} / \mathrm{l}$ derived as median of squares of 1,5 and 10 pixels edge length; (B) Median of squares of $1 \times 1$ to $18 \times 18$ pixels and $95 \%$ confidential interval.
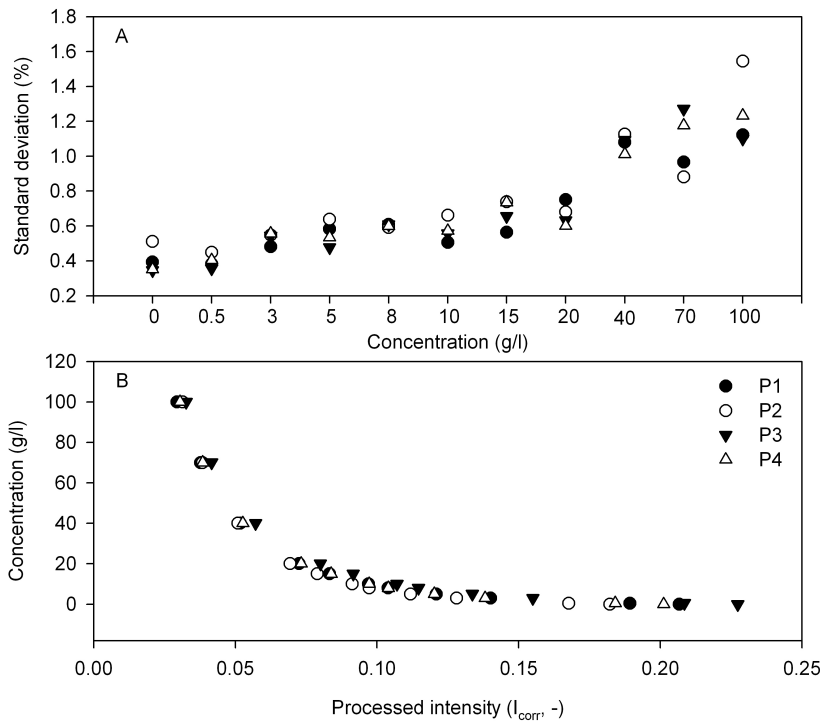

Fig. 5. (A) Standard deviations of calibration images at observation point P1-P4 (Fig. 2); (B) Intensity vs. concentration calibration plot of P1-P4.

3.4 Image calibration and converting of intensities to concentrations (Steps 5 and 6)

In order to relate a given value of intensity to a concentration of salt or dye, calibration runs were made with solutions of predetermined dye-salt concentrations. In the calibration experiment solutions of $0.5,3,5,8,10,15,20,40$, $70,100 \mathrm{~g} / \mathrm{l}$ of saltwater and the dye concentration of the respective solution at a ratio of $1 / 100$ of the salt concentration
Table 2. Calibration parameters of second order exponential function.

\begin{tabular}{lllll}
\hline & $\mathrm{P} 1$ & $\mathrm{P} 2$ & $\mathrm{P} 3$ & $\mathrm{P} 4$ \\
\hline$y 0$ & -0.81727 & -0.99127 & -0.75114 & -0.71146 \\
$A 1$ & 89.4648 & 580.7729 & 376.0868 & 120.6542 \\
$t 1$ & 0.04421 & 0.01295 & 0.01657 & 0.03952 \\
$A 2$ & 355.5046 & 111.7840 & 96.76833 & 417.4293 \\
$t 2$ & 0.01577 & 0.03839 & 0.04733 & 0.01368 \\
$R^{2}$ & 0.99997 & 0.99998 & 0.99998 & 0.99998 \\
\hline
\end{tabular}

were pumped into the tank and photos were taken. Due to the higher ion activity of the saltwater we used salt-dye solutions for calibration. This accounts for possible intensity differences between equally concentrated solutions of dyesaltwater and dye-freshwater. The solutions were pumped into the tank from the bottom of the tank upwards in a sequential order from low-density solutions to high-density solutions. This prevents instabilities and enables a uniform filling of the domain. 50 photos were made of each calibration step. Since we use linear raw data the brightness-corrected intensities (see Sect. 3.2) can be translated directly to concentrations based on intensity vs. concentration standard curves, which have to be derived for each point where concentrations are to be determined. In our experiment, the salt concentration, and not the tracer concentration, is of particular interest. Therefore, we relate intensities of the dye directly to salt concentrations (Fig. 5B), which are just 100 times the dye concentration. The plotted intensities vs. concentrations of observation points P1, P2, P3 and P4 (Fig. 2) in Fig. 5B exhibit a non-linear relationship which agrees with observations by Catania et al. (2008) and Jones and Smith (2005) who used fluorescent dyes. The theoretical relation between intensity and concentration (in the case of transmittance) follows an exponential function. This relation is known as LambertBeer's law and it is valid for monochromatic light and for solutions without porous media. Because this is not the case in our experiments, we tested various functions to relate intensity and concentration (power laws of higher order and exponential functions). A second order exponential function turns out to be the most suitable for relating intensities to concentrations (Eq. 4, Table 2).

$C(t)_{i, j}=A 1_{i, j} \cdot \exp \left(I_{\text {corr }}(t)_{i, j} / B 1_{i, j}\right)+A 2_{i, j} \cdot \exp$
$\left(I_{\text {corr }}(t)_{i, j} / B 2_{i, j}\right)+y 0_{i, j}$

$C(t)_{i, j}$ Concentration at time $t A 1_{i, j}, A 2_{i, j}, y 0_{i, j}, B 1_{i, j}$, $B 2_{i, j}$ Parameters; see Table 2 for values $I_{\text {corr }}(t)_{i, j}$ Brightness corrected intensity at time $t$

Since we need to resolve concentration ranges from 0 to $100 \mathrm{~g} / \mathrm{l} \mathrm{salt}, 1 \mathrm{~g} / \mathrm{l}$ dye concentration proves to be adequate to mark the maximum salt concentration of $100 \mathrm{~g} / \mathrm{l}$. The initial concentration (maximum concentration) of dye must be 


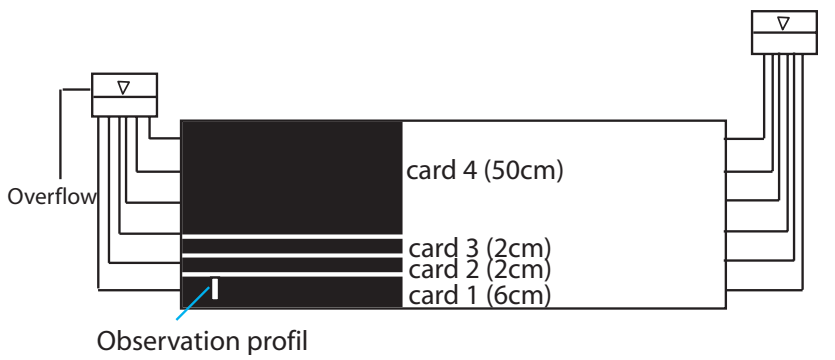

Fig. 6. Setup of the experiment E1. The cards are placed one after another right above each other, the heights of the cards are written in brackets.

high enough so that, even with a large dilution, e.g. $0.5 \mathrm{~g} / \mathrm{l}$ salt (corresponding to $0.005 \mathrm{~g} / \mathrm{l}$ color), the plume can be differentiated optically from the ambient pore water. However, the maximum concentration needs to be below intensity saturation to enable the correct resolution of the high concentrations.

The parameters of the calibration curve had to be derived for each specific observation point (Table 2). Thus, background levelling, as suggested in Schincariol et al. (1993) to compensate the spatially uneven lighting in the tank, was not necessary. This is an advantage because background levelling can reduce the dynamic range and adds noise to the original data (Russ, 1999). Once the parameters of the standard curve are determined, they apply to all experiments if illumination and camera position is constant. This approach offers a computationally effective way to derive the high-resolution time series of concentrations.

\subsection{Impact of lens flare on measured intensities}

The basis of photometry in terms of concentration measurements of fluids is as follows: the brightness of two solutions is of equal intensity during calibration and all stages of the experiment, provided that the solutions are equally concentrated (Arnold et al., 1971). However, this prerequisite could become perturbed due to the effects of lens flare. Rogers (in Newman, 1976) stated: "one of the most usual problems created by white backgrounds is that of flare, caused by excessive reflection from white surface into the camera lens [...]." In porous media experiments, the artificial glass beads cause high reflection intensity and might generate flare effects, which have enough intensity to perturb the measurements. Regions of the photographed domain, which have a high dye concentration, might appear brighter because of the lens flare effects. That causes an underestimation of concentrations. We conducted a series of experiments in order to investigate the impact of lens flare effects on reflected intensity measurements (Table 1 ).

In experiment E1, we successively placed four $70 \mathrm{~cm}$ long black cards one after another at the left side of the front window of our tank as illustrated in Fig. 6. Fifty images were

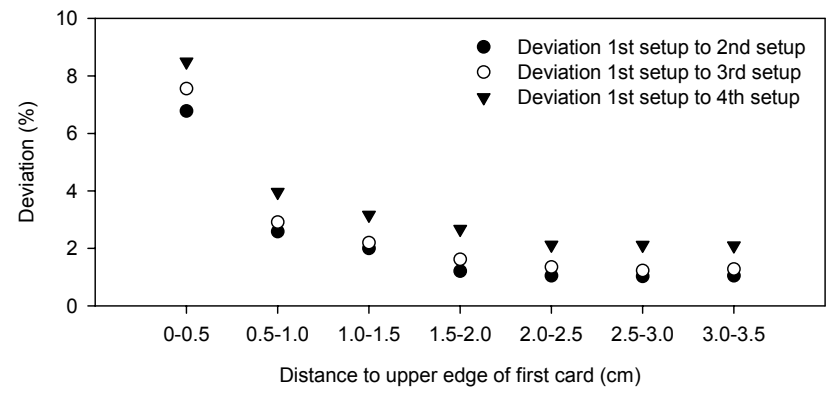

Fig. 7. Deviation (decline) of intensities taken at the profile in Fig. 6 when adding cards above card 1 .

made of each of the 4 steps. Figure 7 shows the decline of intensities along a vertical profile on card 1 (Fig. 6) for the four steps of the experiment. Adding cards 2 to 4 above card 1 decreases the reflection from the porous media above card 1 and therefore the effect of flare was reduced and made card 1 appear darker. The fourth stage of E1 is comparable to the calibration phase of E2 (see Sect. 3.4) when the entire tank was filled with the dyed saltwater and lens flare significantly reduced. However, the dye tracer did not affect the largest portion of the tank during the flow phase of E2, and reflection intensity of the porous media was high. The shape of the salt body during the upconing process of the flow phase of E2 is shown schematically in Fig. 1. Flare effects like in E1 can occur. In order to demonstrate the flare effects in E2 we derived intensities at a point $2 \mathrm{~cm}$ above the ground. The position of this measurement point is equal to the observation hole P1 of E3 (Fig. 2). Concentration profiles showed that the transition zone of the saltwater at the end of the equilibration phase, the second phase of the saltpool experiment, started $3 \mathrm{~cm}$ above the ground. Therefore, the concentration is necessarily $100 \mathrm{~g} / \mathrm{l}$ at $2 \mathrm{~cm}$ above ground. Since the salt body moves upwards to the outlet during the course of the experiment, no concentration changes are expected at this point. The intensities in Fig. 8 show a clear shift from $100 \mathrm{~g} / \mathrm{l}$ calibration to the equilibration phase (approximately $7 \%$ increase in intensity) and from calibration to the flow phase (approximately 3\%). The intensity values decline during the upconing process of the flow phase. With the upconing of the saltwater, the dyed water affects a larger region around the observation point and reflection intensities are reduced. Therefore, the flare effects are reduced and the deviation between calibration intensities for $100 \mathrm{~g} / \mathrm{l}$ and the measured intensities during the flow phase decline to $3 \%$. This effect is comparable to the effects observed in E1 when the black cards are attached above card 1.

Rogers (in Neuman, 1976) suggested: "this problem [of lens flare] is largely overcome [...] and reduced to a minimum by masking the background area so that as little as possible is displayed." Therefore, we attached a mask on the photographed domain with holes $\left(1.5 \times 1.5 \mathrm{~cm}^{2}\right)$ at those 


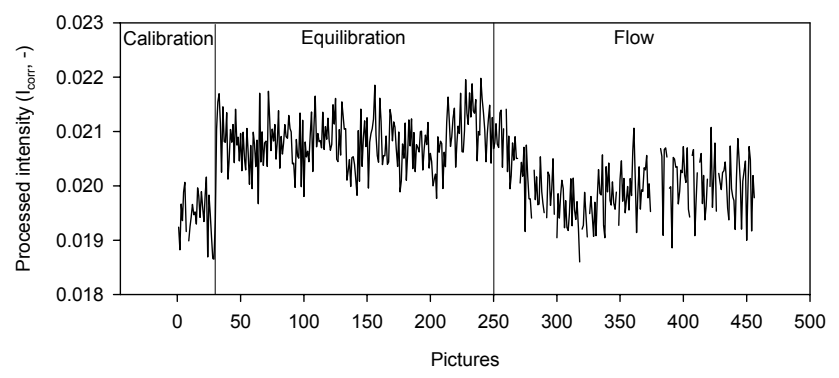

Fig. 8. Intensities of P1 (without mask) where no concentration changes occur during the flow phase of the density flow experiment (constant concentration of $100 \mathrm{~g} / \mathrm{l}$ ).

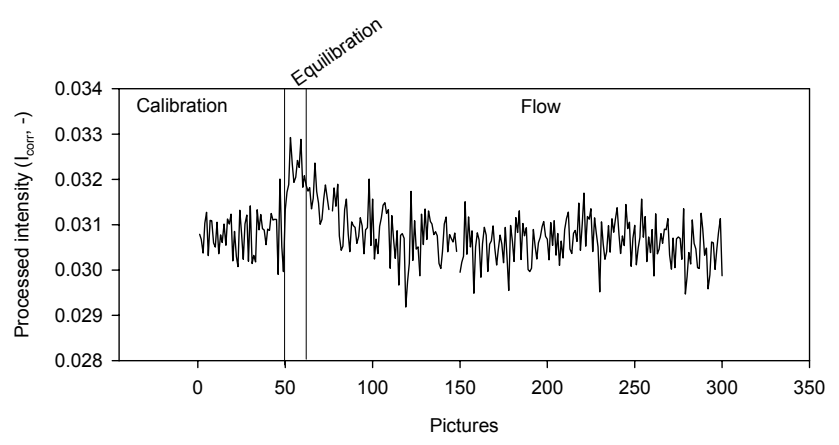

Fig. 9. Intensities of observation point $\mathrm{P} 1$ with mask.

points where we wanted to derive breakthrough curves for the third experiment E3 (Fig. 2). The upconing took place at the left side of the domain below the outlet. Therefore, as many observation points as possible were distributed in or close to the area of the expected saltwater upconing. Figure 9 shows the intensities of P1 during all stages of the experiment. It becomes evident that the effect of lens flare is strongly reduced, especially during the flow phase $(0 \%$ intensity deviation between calibration and flow phase). However, there is still an approximately 3\% deviation between the intensities of the calibration and the equilibration phases of E3. We attribute this effect to the impact of the observation points close to $\mathrm{P} 1$. These were not influenced by the dye and therefore reflection intensity was high and, hence, could cause lens flare effects. During the course of the experiment the salt plume rose and the points in the neighbourhood of P1 appeared darker, which reduced flare effects during the flow phase comparable to the effects observed in E1 and E2. Therefore, the attached mask helps to reduce flare effects.

The experiment E4 was conducted to estimate the error caused by flare effects. Solutions of predetermined 5, 40, and $100 \mathrm{~g} / \mathrm{l}$ (salt+dye) were progressively pumped into the tank from the bottom upward. Seven different filling levels with a horizontally planar salt water front were established so that all observation points in the same horizontal line were fully immerged in the solution. In order to estimate the impact

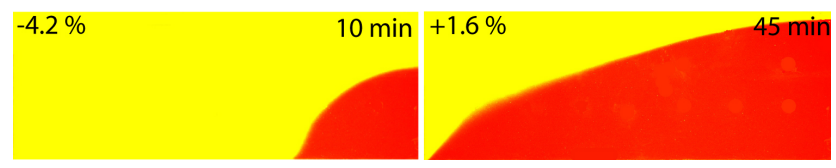

Fig. 10. Mass balance analysis of E5 after $10 \mathrm{~min}$ and $45 \mathrm{~min}$.

of lens flare we derived intensities at all observation points visible in Fig. 2 (not only P1-P4) for all filling levels of the experiment of the three solutions and analyzed their development during the course of the experiment. E4 revealed that the error depends on (i) the concentration of the solution, and the error increases with increasing concentration; and (ii) the intensity of the neighboring observation points, while the error decreases with decreasing intensity. The maximal deviation of intensities for the $100 \mathrm{~g} / \mathrm{l}$ solution in E4 amounted to $3 \%$. This experiment shows only 1-D vertical movement of the brine, and 2-D plume shapes such as in the saltpool experiments E2 and E3 do not occur. Therefore, we used the results of E4 only to estimate the error caused by lens flare effects, and not to correct the measured data. We assumed a maximal error of $3 \%$ of overestimation (compared to calibration) of the intensities during the flow phase caused by lens flare effects for E3 with the attached mask.

\subsection{Error assessment}

In order to estimate the precision of the photometric method, we calculated the standard deviation of the calibration images for each calibration step (Fig. 5A). The maximum deviation, which we consider as the accuracy of the measured intensities, occurs for salt concentration of calibration steps $70 \mathrm{~g} / \mathrm{l}$ or $100 \mathrm{~g} / \mathrm{l}$. From the above discussion, we know that there is an additional error of $+3 \%$ in the measured intensities due to the lens flare effect. Therefore, the concentrations are given with confident limits (see Fig. 16). The lower boundary is calculated from the processed intensity $I_{\text {corr }}$ plus standard deviation $(\%)$, whereas for the upper boundary $I_{\text {corr }}$ minus $3 \%$ and standard deviation is used to derive concentrations. The intensities are converted to concentrations using the second order exponential equation (Eq. 4). This propagates the intensity error non-linearly to the concentration error. The mass balances in experiment E5 can also be calculated to test the performance of the image analysis approach based on the given mass of salt entering the domain and the mass determined by image analysis at different time steps (Fig. 10). The image analysis takes into account the porosity and the depth of the tank. The impact of lens flare becomes more pronounced if a small portion of the domain is filled with the dark solution. If the tank is filled with the dark solution the effect should be reduced. In E5, the dye-salt solution enters the domain over one inlet at the edge of the tank with a constant well-known flow rate. The concentration is $100 \mathrm{~g} / \mathrm{l}$ of $\mathrm{NaCl}$. An image is taken every $30 \mathrm{sec}$ and we analyzed the 


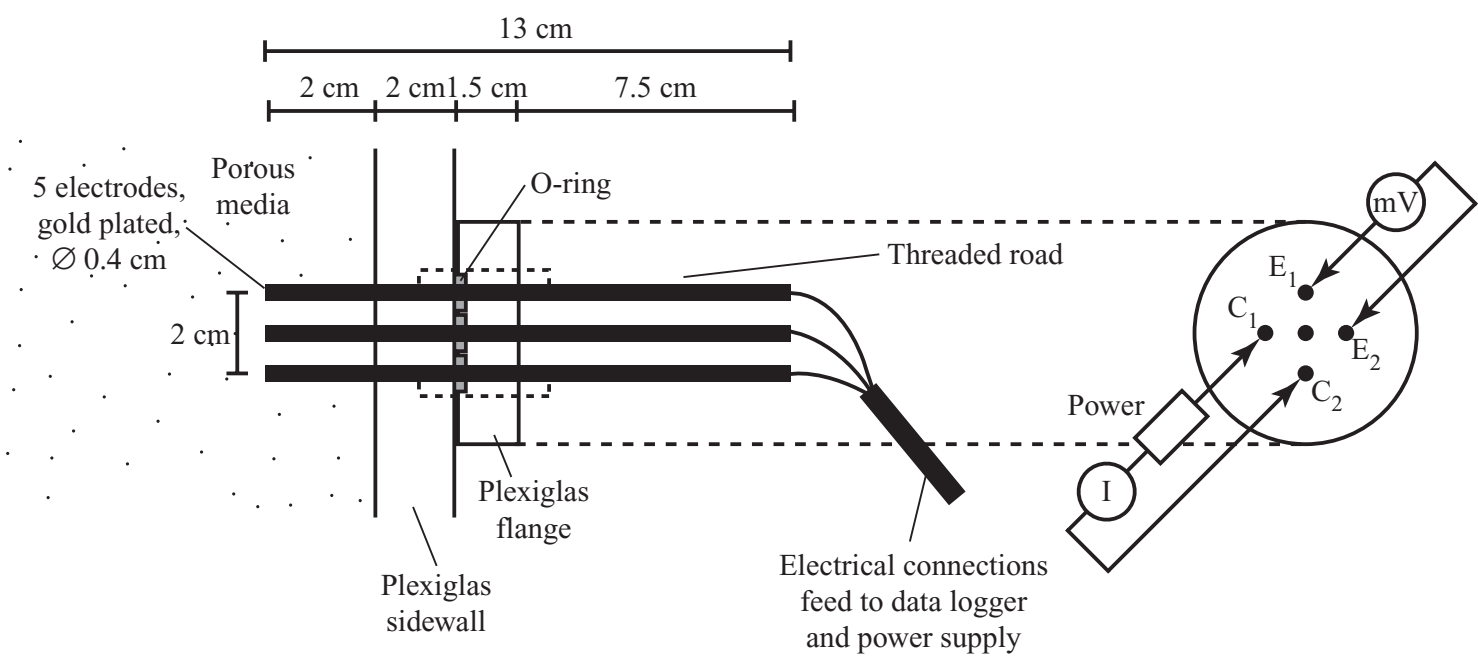

Fig. 11. Electrode array with two current electrodes $\left(C_{1}\right.$ and $\left.C_{2}\right)$, two potential electrodes $\left(E_{1}\right.$ and $\left.E_{2}\right)$ and grounding in the center of the array.

images after 10 and 45 minutes. Figure 10 shows the spatial distribution of the saltwater (red). The $10 \mathrm{~min}$ image delivers an underestimation of the total mass of $-4.2 \%$ compared to the mass entering the domain. This is within the range of expected lens flare error. After $45 \mathrm{~min}$, the bright region of the tank is significantly reduced and the mass error accounts for $1.6 \%$ indicating that the method overestimates the total mass. On the basis of this analysis, the calibration parameters of Eq. 4 can be considered reliable, and possible errors can be explained by the impact of lens flare.

\section{Concentration determination with resistivity measur- ing cells (RMC)}

\subsection{Technical setup of the RMC system}

Each RMC consists of five gilded stainless steel electrodes (diameter $0.4 \mathrm{~cm}$, length $13 \mathrm{~cm}$, Fig. 11). We used gilded electrodes, because uncontrollable fluctuations in the resistivity measurement occur with ungilded stainless steel electrodes. The electrodes are equally spaced $1 \mathrm{~cm}$ around the center electrode and mounted on a Plexiglas flange. They are installed perpendicular to the flow direction through the back wall of the tank. Figure 12 shows the schematic construction plan of the entire RMC system. The system involves measuring the output voltage between the electrodes $E_{1}$ and $E_{2}$ while electrical current is caused to flow through the porous media between the outer pair of electrodes $C_{1}$ and $\mathrm{C}_{2}$. The grounding electrode is in the center. The measuring principle is based on a 4-electrode resitivity measurement technique, where the current is supplied through a very precisely regulated sinusoidal oscillator signal. The measured
AC voltage is rectified through a synchronous demodulator in phase to the oscillator. The resulting DC output voltage was recorded utilizing a micro-logger and a relais based multiplexer to measure all the RMCs sequentially. An alternating current was applied in all measurements to minimize polarization within the cell and to prevent movement of the ions due to the presence of a voltage potential.

Since the measurement has to cover a wide range of voltage values due to the high salt concentrations, two different input voltages are used at each time step. This enables measurements to be made in two measurement ranges, one for the high concentration, referred to as MR1, and the other for low concentrations, MR2. As this measuring method is sensitive to temperature variations, temperature sensors (YSI 4006; accuracy $\pm 0.01^{\circ} \mathrm{C}$ ) are also inserted into the porous medium.

\subsection{Electrode arrays}

Various electrode arrays were tested in preliminary experiments in a small container $\left(35 \times 16 \times 9 \mathrm{~cm}^{3}\right)$ filled with saturated porous media. Figure 13 shows the three investigated arrays. In array 1 all electrodes are uniformly placed in a line with the grounding electrode in the center. Array 2 is a cross-placement of $\mathrm{C}_{1,2}$ (current electrodes) and $\mathrm{E}_{1,2}$ (potential electrodes) while in array 3 input and output electrodes are placed in an adjacent position (parallel). In preliminary tests to evaluate the best array setup, the three different arrays were also rotated clockwise to study the influence of flow direction of the saltwater front. Array 1 was rotated clockwise by $90^{\circ}$, array 3 was rotated clockwise by $90^{\circ}, 135^{\circ}, 180^{\circ}$ and $315^{\circ}$. A total of 4 tests were carried out. The setups were evaluated by measuring a successively rising saltwater 


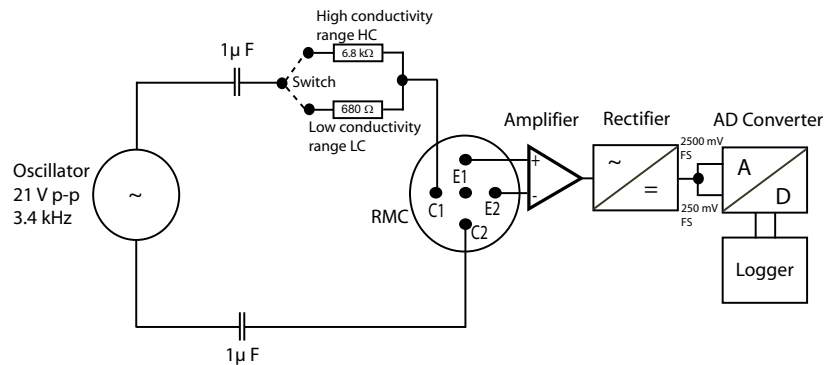

Fig. 12. Construction plan of the RMC system.
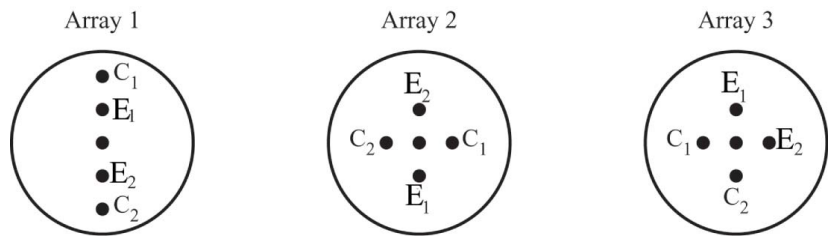

Fig. 13. Evaluated sets of electrode arrays.

front in the small experimental tank. Figure 14 shows the measured breakthrough curves of output voltages of the 8 different arrangements.

The breakthrough curves of array 1 and its clockwise rotated (by $90^{\circ}$ ) setup clearly show the dependence of these alignments on the flow direction of the salt front. There is a temporal shift between both curves, and array 1 even shows a plateau of the curve. Array 2 delivers no analyzable breakthrough curve. In this setup, both electrodes should detect the same voltage under homogeneous conditions. The fact that a voltage change is visible might be due to local concentration differences or asymmetric manufacturing of the RMC. Array 3 and its rotated setups deliver curves less dependent on the flow direction of the front and a realistic shape of the curve is observed. As the result of these findings, we chose array 3 for measuring the resistivity.

\subsection{Calibration of the RMC}

The resistivity measured between two electrodes is a function of the porous material, porosity, temperature, geometrical arrangement of the electrodes, state of gold coating and the ion concentration (Telford et al., 1990). During an experiment, all factors apart from the ion concentration are kept constant and the measured resistivity can be related to the concentration of the solution surrounding the electrodes. This relationship has to be determined via calibration experiments as described in Sect. 3.4. The output voltages of the two measuring ranges, MR1 and 2, are correlated with the concentrations using specifically fitted power laws for each RMC and measurement range. Figure 15 shows, by way of example, the calibration curves for MR1 and 2 of the RMC located at observation point P2 (Fig. 2).

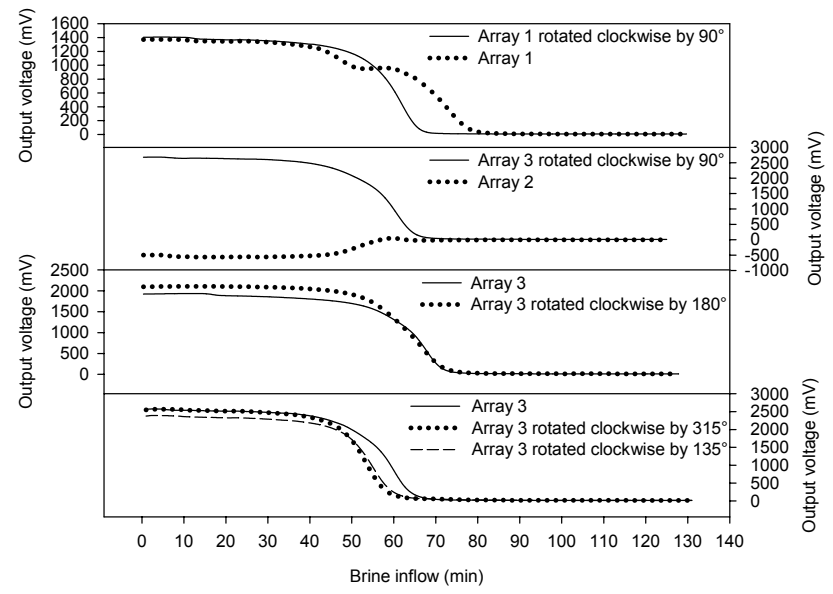

Fig. 14. Measured breakthrough curves of output voltage $(\mathrm{mV})$ for the different sets of electrodes and their clockwise-rotated arrangements.

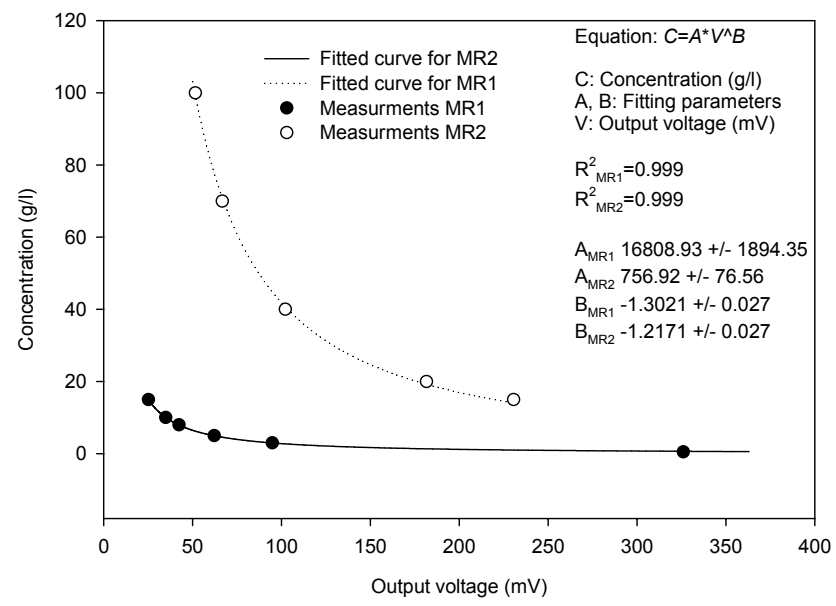

Fig. 15. Calibration curves for the RMC at $\mathrm{P} 2$.

The errors associated with the concentration consist of two parts: measurement errors and parameter errors associated with the calibration curves. The measurement errors are small and the maximum error found during the calibration experiment was $+/-0.5 \%$. The errors associated with the calibration curve are within the range of -1.5 to $+2.5 \%$. Since the calculated error is small we do not show error bars in Fig. 16. Typically, systematic errors are caused by temperature fluctuations. However, these can be minimized for laboratory experiments due to controlled room and water temperatures.

\section{Comparison of the two methods}

For direct comparison of the RMC method and the image analysis method we derived concentrations from the images at the position of the centre electrode of the RMC at observation points $\mathrm{P} 2$ and P3 (Fig. 2). The comparison reveals that 
both independent methods reproduce the expected concentrations of $100 \mathrm{~g} / \mathrm{l}$ at P2 when the saltwater rises, although the RMC shows a slightly earlier reaction than the image analysis approach, which can be explained by the larger measurement volume of the RMC (Fig. 16).

However, if the electrode is placed within the transition zone, where steep concentration gradients exist, the two methods differ significantly due to the different measurement volumes, as is the case at P3 (Fig. 16). The RMC measures an apparent resistivity of the environment around the electrodes. Thus, it is not a point measurement but an integral value. The measurement volume strongly depends on the ion concentration distribution and on the resistivity contrast during the experiment. Therefore, the measurement volume is not constant throughout the course of the experiment. This problem is the major drawback of the RMC method especially for measuring points within the transition zone, where a concentration gradient occurs within the measuring volume of the RMC. However, especially for points such as P2 where the electrodes are surrounded by a uniform concentration, this problem is of minor importance. A numerical simulation of the resistivity field, the current density and of the voltage at the electrodes for heterogeneous resistivity distributions could enable the determination of the measurement volume. This work was beyond the scope of this study but we highly recommend further investigation in this field.

\section{Conclusions}

In this study we present a photometric methodology, which enables effective computation of high-resolution time series of plume concentrations. The method is capable of treating a large number of images since only one optimization of the intensity vs. concentration standard curve is required. Once the parameters of this curve are known for the observation point they can be used for a series of experiments, if the camera position and the tank position do not change and the images fit on a pixel-by-pixel basis. Beside the breakthrough curves contour lines of concentration can be determined. The resolution of the observation point is crucial for the precision of the measured intensity values. Therefore, we suggest a statistical analysis for each experimental setup to determine the adequate resolution. A major source of error is the impact of lens flare. High reflections of bright regions of the photographed domain, which are not influenced by the dye, could generate flare effects that increase the measured intensity of dark, dye-influenced regions of the tank. This leads to an underestimation of the concentration. Attaching a mask at the front window of the tank, which reduces the percentage of bright regions, can minimize this effect. However, information is available in this case only at the predetermined points, i.e., the observation holes in the mask. Since the mask itself influences the brightness of the observation holes, both the calibration and the flow experiments have to

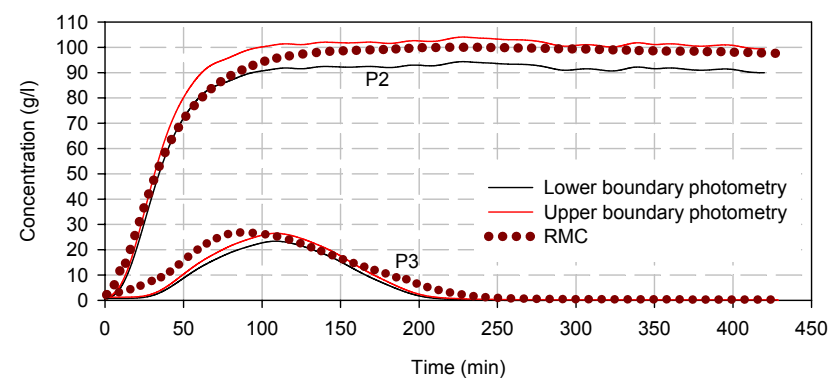

Fig. 16. Breakthrough curves of $P 2$ (100 g/l) and P3 (max. $30 \mathrm{~g} / \mathrm{l}$ ) derived from electrodes and from photometric method. The solid lines represent the range of error of the photometric method.

be conducted using the same setup with the mask. This minimizes the flexibility of the experiment in order to run different experiments with changing boundary conditions and therefore with different plume patterns.

Nevertheless, the precision of the measurement is high even without the mask. A successive filling of the tank with predetermined concentrations of salt-dye solutions enables an estimation of the error caused by flare effects. The successive filling gradually reduced the percentage of bright regions and therefore decreased the impact of flare effects at the observation points. The deviation between the measured intensity of the entirely filled tank and the partly filled tank was determined as maximal error.

In this study, the light reflection technique was used instead of the transmission technique because:

1. The back of the tank holds devices to measure resistivity, temperature and pressure;

2. Applicability and limitations of the widely used reflection method need to be assessed.

For future research, we suggest a direct comparison of the light reflection and the transmission techniques.

The resistivity measurement system provides highly precise measurements of output voltages, which can be translated into concentrations using a power law. The major perturbations of the measurement are temperature changes, which can be avoided in laboratory scale experiments. However, the main drawback of the resistivity method is the unknown measurement volume. For the evaluation of numerical codes it is crucial to know the exact measurement volume, especially in an intermediate scale experiment where significant variations in concentrations occur, even over a few centimetres. Therefore, we consider the image analysis approach as more suitable for deriving breakthrough curves for benchmarking numerical codes. The electrodes, however, can be used to cross-check the results of the image analysis method at points of uniform concentration distribution. Further, the methodology is useful in 3-D experiments of large tank volumes to determine concentrations at 
points within the tank. A numerical analysis of the measurement volume of the RMC system could help to improve the applicability of the technique for detailed laboratory-scale flow experiments. Regarding hydrogeological field observations, the RMC method could be further developed for multilevel monitoring of electrical conductivities in the context of highly saline groundwaters mixing with groundwaters of low salinity. This is of special interest in active evaporite subrosion and/or forced groundwater circulation systems (active pumping). Resistivity tomography could be tested and compared with the imaging method - viewing the results of the imaging method as "true" system behaviour.

Acknowledgements. The authors are grateful to Claude Veit (IMFS, Strasbourg) who constructed the Plexiglas flow tank and to Prof. Wieland for support during the development of the RMC method. Lukas Rosenthaler of the Imaging and Media Lab, University of Basel contributed significantly to the image analysis method. We acknowledge the valuable comments of two anonymous referees and of the editor. This study was financed by the Schweizer Nationalfond 200020-109200.

Edited by: C. Hinz

\section{References}

Arnold, C., Rolls, P., and Stewart, J.: Applied photography, The Focal Press, ISBN 0-240-50723-1, 1971.

Barth, G. R., Illangesekare, T. H., Hill, M. C., and Rajaram, H.: A new tracer-density criterion for heterogeneous porous media, Water Resour. Res., 37, 21-31, 2001.

Catania, F., Massabo, M., Valle, M., Bracco , G., and Paladino, O.: Assessment of quantitative imaging of contaminant distributions in porous media, Exp Fluids 44, 167-177, 2008.

Corapcioglu, M. Y., Chowdhury, S., and Roosevelt, S. E.: Micromodel visualization and quantification of solute transport in porous media, Water Resour. Res. 33, 11, 2547-2558, 1997.

Danquigny, C. Ackerer, P., and Carlier, J. P.: Laboratory tracer tests on three-dimensional reconstructed heterogeneous porous media, J. Hydrol., 294(1-3), 196-212 Sp. Iss. SI JUL 15, 2004.

Detwiler, R. L., Rajaram, H., and Glass, R. J.: Solute transport in variable-aperture fractures: An investigation of the relative importance of Taylor dispersion and macrodispersion, Water Resour. Res. 36, 7, 1611-1625, 2000.

Goswami, R. and Clement, P.: Laboratory-scale investigation of saltwater intrusion dynamics, Water Resour. Res., 43, W04418, doi:10.1029/2006WR005151, 2007.

Hassanizadeh, S. M. and Leijnse, A.: A non-linear theory of highconcentration-gradient dispersion in porous media, Advances in Water Resour., 18(4), 203-215, 1995.

Huang, W., Smith, C. C., Lerner, D. N,. Thornton, S. F., and Oram A.: Physical modelling of solute transport in porous media: evaluation of an imaging technique using UV excited fluorescent dye., Water Res 3(7), 1843-1853, 2002.
Jones, E. H. and Smith, C. C.: Non-equilibrium partitioning tracer transport in porous media: 2-D physical modelling and imaging using a partitioning fluorescent dye, Water Res 39(2)0, 50995111, 2005.

McNeil, J. D., Oldenborger, G. A., and Schincariol, R. A.: Quantitative imaging of contaminant distributions in heterogeneous porous media laboratory experiments. J. Contam. Hydrol., 84, 36-54, 2006

Oostrom, M., Dane, J. H., Guven, O., and Hayworth, J. S.: Experimental investigation of dense solute plumes in an unconfined aquifer model, Water Resour. Res., 28, 2315-2326, 1992.

Oostrom, M., Hofstee, C., Lenhard, R. J., Wietsma, and T. W.: Flow behavior and residual saturation formation of liquid carbon tetrachloride in unsaturated heterogeneous porous media, J. Contam. Hydrol., 64, 93-112, 2003.

Oswald, S., Kinzelbach, W., Greiner, A., and Brix, G.: Observation of flow and transport processes in artificial porous media via magnetic resonance imaging in three dimensions, Geoderma 80 , 417-429, 1997.

Oswald, S. and Kinzelbach, W.: Three-dimensional physical benchmark experiments to test variable-density flow models, J. Hydrol., 290, 22-42, 2004.

Rahman, A., Jose, S., Nowak, W., and Cirpka, O.: Experiments on vertical transverse mixing in a large-scale heterogeneous model aquifer, J. Contam. Hydrol., 80, 130-148, 2005.

Rogers, G.: Photography in paediatrics, Published in photographic techniques in scientific research, Volume 2, edited by Newman, A., Academic Press, ISBN 012 517960, 1976.

Russ, J. C.: The image processing handbook, 3rd edn. CRC, Boca Raton, 1999

Schincariol, R. A., Herderick, E. E., and Schwartz, F. W.: On the application of image analysis to determine concentration distributions in laboratory experiments, J. Contam. Hydrol., 12, 197215, 1993.

Silliman, S. E., Zheng, L., and Conwell, P.: The use of laboratory experiments for the study of conservative solute transport in heterogeneous porous media, Hydrogeol. J., 6, 166-177, 1998.

Simmons, C. T., Pierini, M. L., and Hutson, J. L.: Laboratory investigation of variable-density flow and solute transport in unsaturated-saturated porous media, Transp. Porous Media 47, 15-244, 2002.

Swartz, C. H. and Schwartz, F. W.: An experimental study of mixing and instability development in variable-density systems, J. Contam. Hydrol., 34, 169-189, 1998.

Telford, W. M., Geldart, L. P., and Sheriff, R. E.: Applied geophysics, - 2nd edition, Cambridge University Press, ISBN 0-52133938-3, 1990.

Theodoropoulou, M. A., Karoutsos, V., Kaspiris, C., and Tsakiroglou, C. D.: A new visualization technique for the study of solute dispersion in model porous media, J. Hydrol., 27(1-4), 176-197, 2003.

Wildenschild, D. and Jensen, K. H.: Laboratory investigations of effective flow behavior in unsaturated heterogeneous sands, Water Resour. Res., 35, 17-27, 1999. 